\title{
Forkhead-box A1 suppresses the progression of endometrial cancer via crosstalk with estrogen receptor $\alpha$
}

\author{
JINGYUN WANG ${ }^{1,2}$, WEI BAO ${ }^{2}$, MEITING QIU ${ }^{1,2}$, YUN LIAO $^{1,2}$, QI CHE $^{1,2}$, \\ TINGTING YANG ${ }^{1,2}$, XIAOYING HE ${ }^{2}$, HAIFENG QIU ${ }^{1,2}$ and XIAOPING WAN ${ }^{1}$
}

\begin{abstract}
${ }^{1}$ Department of Obstetrics and Gynecology, Shanghai First People's Hospital Affiliated to Shanghai Jiao Tong University, School of Medicine; ${ }^{2}$ Department of Obstetrics and Gynecology, International Peace Maternity and Child Health Hospital Affiliated to Shanghai Jiao Tong University School of Medicine, Shanghai, P.R. China
\end{abstract}

Received November 15, 2013; Accepted December 16, 2013

DOI: $10.3892 /$ or.2014.2982

\begin{abstract}
Mechanisms governing the function of Forkheadbox A1 (FOXA1), a member of the FOX class of transcription factors, have been extensively studied. However, little is known about the activities and expression pattern of FOXA1 in endometrial cancer (EC). In the present study, we investigated the level of FOXA1 in multiple human EC cell lines and clinical samples by immunohistochemistry, qRT-PCR and Western blot analysis. FOXA1 overexpression was observed in estrogen receptor $(\mathrm{ER}) \alpha$-positive $\mathrm{EC}$ cell lines $(\mathrm{P}=0.0048)$. In endometrial tissues, FOXA1 was significantly upregulated in both normal endometrium and well-differentiated endometrial cancer tissues $(\mathrm{P}<0.001)$. Functional analyses of FOXA1 were evaluated by MTT, plate colony formation and Transwell assay. The results revealed that forced expression of FOXA1 inhibited EC cell proliferation, whereas FOXA1 depletion promoted cell viability and was associated with tumorigenesis. The nude mouse tumor xenograft assay also confirmed that ablation of FOXA1 expression promoted cell proliferation. Furthermore, we found that knockdown of FOXA1 decreased the expression of ER $\alpha$, and FOXA1 interacted with this receptor in the EC cell lines. Collectively, these experiments suggest that FOXA1 is a tumor suppressor in $\mathrm{EC}$ and has a possible interaction with $\mathrm{ER} \alpha$.
\end{abstract}

\section{Introduction}

Endometrial cancer (EC) is one of the most common gynecological malignancies, with an escalating number of new

Correspondence to: Dr Xiaoping Wan, Department of Obstetrics and Gynecology, Shanghai First People's Hospital Affiliated to Shanghai Jiao Tong University, School of Medicine, 100 Haining Road, Shanghai 200080, P.R. China

E-mail: wanxp@sjtu.edu.cn

Abbreviations: EC, endometrial cancer; FOXA1, Forkhead-box $\mathrm{A} 1 ; \mathrm{ER} \alpha$, estrogen receptor $\alpha$; TFs, transcription factors

Key words: endometrial cancer, Forkhead-box A1, estrogen receptor $\alpha$ cases and an increasing mortality rate. In the US, 49,500 new cases of EC will be diagnosed in 2013, and 8,200 deaths are expected (1). Accurate regulation of complex transcriptional programs is central to the progression of EC. Forkhead-box A1 (FOXA1) a member of the FOX family of transcription factors (TFs) that comprises at least 40 members (2), was originally identified for its transcriptional role in early liver and pancreas development (3). At present, FOXA1 is mainly known as a 'pioneer factor', which can occupy distal regulatory enhancers and alter the chromatin accessibility for subsequent recruitment of collaborating TFs, instead of directly promoting transcription activation $(4,5)$. However, the functional role of FOXA1 in EC remains unclear.

Estrogen receptor $\alpha(\mathrm{ER} \alpha)$ is a ligand-activated transcription factor that belongs to the nuclear receptor superfamily (6), and regulates estrogenic action in the female reproductive tract. Upon estrogen stimulation, ER $\alpha$ binds at numerous genomic loci to promote the transcriptional program (7), while several coregulators are simultaneously recruited to the chromosomal loops to facilitate the estrogenic transcriptional response (8-11). Thus, ER $\alpha$ is an ideal target for etiologyspecific therapy in EC. Decreased or absent expression of ER $\alpha$ is regarded to be accompanied by disease progression, but the underlying mechanism is still unclear (12).

A number of studies have reported FOXA1 upregulation in several types of human cancers, including breast cancer, in which the expression patterns of FOXA1 are strikingly similar to that of $\mathrm{ER} \alpha$ (13). We speculated that in endometrial cancer, a disease also closely related to hormone and hormone receptors, FOXA1 may play a potential role in cancer progression and may regulate the transcriptional activation of ER $\alpha$. In the present study, we investigated the level of FOXA1 in multiple human EC cell lines and clinical samples. The expression of FOXA1 in normal endometrial and EC tissues was analyzed and correlated with clinicopathological parameters. The regulatory roles of FOXA1 were investigated both in vitro and in vivo. These experiments suggest that FOXA1 is a tumor suppressor in EC. Furthermore, our results increased our understanding of the ER $\alpha / F O X A 1$ relationship, and we propose that FOXA1 may act as a regulator of the ER signaling pathway in EC cell lines. 


\section{Materials and methods}

Tissue selection and patient information. Paraffin sections from 74 EC tissues were obtained from patients who underwent initial hysterectomy at the International Peace Maternity and Child Health Hospital of the China Welfare Institute, which is affiliated to Shanghai Jiao Tong University School of Medicine, from December 2009 to November 2012. The stages and histological grades of these tumors were established according to the criteria of the International Federation of Gynecology and Obstetrics (FIGO) (14). Sixty-four normal endometrial samples were obtained from patients who underwent hysterectomy to treat other diseases such as adenomyosis or myoma. Twelve atypical hyperplasia tissues were prepared from patients who underwent hysteroscopic examination for the reason of irregular bleeding. Following excision, tissue samples were immediately frozen in liquid nitrogen and stored at $-80^{\circ} \mathrm{C}$ until RNA extraction. Formalin fixed paraffinembedded (FFPE) tissues, matching the frozen cases, were retrieved for IHC analysis. Pathological diagnoses of endometrial lesions were carried out by two gynecologic pathologists based on World Health Organization classifications.

This study was approved by the Human Investigation Ethical Committee of International Peace Maternity and Child Hospital Affiliated Shanghai Jiao Tong University. The samples of normal endometrial tissues, endometrial carcinoma and breast cancer were collected after written informed consent from the patients.

Immunohistochemical analysis. All samples were prepared and analyzed with the Histostain-Plus kit (rabbit) (MRBiotech, Emeryville, CA, USA) according to the manufacturer's protocol. After dewaxing and hydration, 4- $\mu \mathrm{m}$ sections from FFPE tissue were treated by boiling in sodium citrate $(\mathrm{pH} 6.0)$ for $20 \mathrm{~min}$ for antigen retrieval, and endogenous peroxidase activity was blocked by incubation in $0.3 \% \mathrm{H}_{2} \mathrm{O}_{2}$ in methanol for $10 \mathrm{~min}$. Nonspecific binding of antibodies was blocked with serum for $15 \mathrm{~min}$. The slides were then incubated with rabbit polyclonal anti-FOXA1 antibody (1:200; Abcam, Cambridge, MA, USA) for $24 \mathrm{~h}$ at $4^{\circ} \mathrm{C}$. The sections were incubated with a biotinylated secondary antibody (MRBiotech). Then the sections were treated by an horseradish peroxidase-conjugated avidin-biotin complex. Following the manufacturer's instructions, samples were detected by DAB to ensure the intensity of FOXA1 expression. We used the methods of Badve et al to score the staining intensity (15). Briefly, the percentage of staining was classified as ' 0 ' when there was no nuclear expression, ' 1 ' for up to $10 \%$ positive tumor nuclei, ' 2 ' for $11-20 \%$ and continuing in the same manner until a maximum score of ' 10 '. Intensity was scored as '+,' '++', and ' +++ ' for weak, moderate, and strong staining, respectively. The percentage $(\mathrm{P})$ and intensity (I) of nuclear expression were multiplied to generate a numerical score $(\mathrm{S}=\mathrm{P} \times \mathrm{I})$. As a positive control, a section of breast cancer tissue was immunostained with anti-FOXA1 in the same manner. As a negative control, phosphate-buffered saline (PBS) was used to replace the primary antibody. ER $\alpha$ expression status was confirmed in the same manner. Clinical and pathological data relating to the clinical samples are presented in Table I.
Table I. Correlation of FOXA1 expression with clinicopathological parameters of the endometrial carcinoma cases.

\begin{tabular}{|c|c|c|c|}
\hline \multirow[b]{2}{*}{ Characteristics } & \multirow[b]{2}{*}{ Case $(n)$} & $\begin{array}{c}\text { FOXA1 } \\
\text { histoscores }\end{array}$ & \multirow[b]{2}{*}{$\mathrm{p}$-value } \\
\hline & & Means \pm SD & \\
\hline \multicolumn{4}{|l|}{ Age (years) } \\
\hline$\leq 55$ & 31 & $11.03 \pm 4.658$ & \multirow[t]{2}{*}{$0.411^{\mathrm{b}}$} \\
\hline$>55$ & 43 & $10.26 \pm 5.741$ & \\
\hline \multicolumn{4}{|l|}{ Histology } \\
\hline Endometrioid & 62 & $11.76 \pm 4.827$ & \multirow[t]{2}{*}{$<0.001^{\mathrm{b}}$} \\
\hline $\begin{array}{l}\text { Non-endometrioid } \\
\text { (serous/clear) }\end{array}$ & 12 & $4.50 \pm 2.939$ & \\
\hline \multicolumn{4}{|l|}{ FIGO stage } \\
\hline Early (I-II) & 65 & $11.35 \pm 5.094$ & \multirow[t]{2}{*}{$0.001^{b}$} \\
\hline Late (III-IV) & 9 & $5.00 \pm 2.872$ & \\
\hline \multicolumn{4}{|l|}{ Histological grade } \\
\hline Grade 1 & 37 & $14.27 \pm 3.437$ & \multirow[t]{3}{*}{$<0.0001^{\mathrm{a}}$} \\
\hline Grade 2 & 18 & $8.67 \pm 4.498$ & \\
\hline Grade 3 & 19 & $5.21 \pm 2.974$ & \\
\hline \multicolumn{4}{|c|}{ Lymph node metastasis } \\
\hline Positive & 7 & $4.43 \pm 2.992$ & \multirow[t]{2}{*}{$0.002^{b}$} \\
\hline Negative & 67 & $11.22 \pm 5.075$ & \\
\hline \multicolumn{4}{|l|}{ Myometrial invasion } \\
\hline$\leq 1 / 2$ & 47 & $12.23 \pm 5.117$ & \multirow[t]{2}{*}{$0.001^{\mathrm{b}}$} \\
\hline$>1 / 2$ & 27 & $7.70 \pm 4.340$ & \\
\hline \multicolumn{4}{|l|}{ ER expression } \\
\hline Positive & 58 & $12.00 \pm 4.761$ & \multirow[t]{2}{*}{$<0.0001^{\mathrm{b}}$} \\
\hline Negative & 16 & $5.44 \pm 3.777$ & \\
\hline
\end{tabular}

Significance of difference ( $\mathrm{p}$-value) between categories was analyzed by ${ }^{a}$ Kruskal-Wallis rank test and ${ }^{\mathrm{b}}$ Mann-Whitney $\mathrm{U}$ test, respectively. p-values indicating statistically significant results are in bold print.

Cell culture. Human endometrial cell lines (Ishikawa, RL95-2, HEC-1B and AN3CA) were obtained from the Chinese Academy of Sciences Committee Type Culture Collection cell bank. The cells were grown in Dulbecco's modified Eagle's medium:Nutrient Mixture F-12 (DMEM/F12) supplemented with $10 \%$ fetal bovine serum (FBS) and $1 \%$ penicillin/streptomycin (Gibco, Auckland, New Zealand) in a humidified atmosphere of $5 \% \mathrm{CO}_{2} / 95 \%$ air at $37^{\circ} \mathrm{C}$.

RNA extraction and quantitative real-time PCR. Total RNA was prepared from the EC cell lines using TRIzol RNA isolation reagents (Invitrogen, Carlsbad, CA, USA). The cDNA was generated with the Prime Script RT reagent kit (Takara Inc., Otsu, Japan). A 50- $\mu 1$ PCR amplification of single-strand cDNA was performed with 40 cycles of denaturation $\left(94^{\circ} \mathrm{C}\right)$ for $60 \mathrm{sec}$, annealing $\left(55^{\circ} \mathrm{C}\right)$ for $30 \mathrm{sec}$, and elongation $\left(72^{\circ} \mathrm{C}\right)$ for $30 \mathrm{sec}$ using SYBR Premix Ex Taq (Takara Inc.). The primer sequences are listed in Table II. For all the data, values 
Table II. Primer sequences for real-time PCR analysis.

mRNA Primer sequence

FOXA1 Forward 5'-AGGTGTGTATTCCAGACCCG Reverse 5'-TTGACGGTTTGGTTTGTGTG

ER $\alpha$ Forward 5'-TGATTGGTCTCGTCTGGCG Reverse 5'-CATGCCCTCTACACATTTTCCC

pS2 Forward 5'-GTGTCACGCCCTCCCAGT Reverse 5'-GGACCCCACGAACGGTG

GREB1 Forward 5'-CAAAGAATAACCTGTTGGCCCTGC Reverse 5'-GACATGCCTGCGCTCTCATACTTA

ACTB Forward 5'-CAGCCATGTACGTTGCTATCCAGG Reverse 5'-AGGTCCAGACGCAGGATGGCATG

on the $\mathrm{y}$-axis employed the $2^{-\mathrm{ACt}}$ method. Data were obtained in triplicate from three independent experiments.

Western blot analysis. Cells were lysed for total protein extraction using ProteoJET Mammalian Cell Lysis Reagent (MBI Fermentas, Ontario, Canada) including a protease inhibitor cocktail (Roche Diagnostics, Basel, Switzerland). Protein $(80 \mu \mathrm{g})$ was loaded onto precast $4 \%$ stacking, $10 \%$ Tris-glycine gels and separated by gel electrophoresis. Proteins in the gels were then transferred to a polyvinylidene difluoride (PVDF) membrane. After transfer, membranes were blocked with 5\% bovine serum albumin (BSA)/phosphate-buffered saline (PBS) for $3 \mathrm{~h}$. The membranes were incubated with primary antibodies at $4^{\circ} \mathrm{C}$ overnight. The membranes were then incubated with peroxidase-linked secondary antibody $(1: 10,000$; Epitomics, Burlingame, CA, USA) for $2 \mathrm{~h}$ at room temperature. The blotted proteins were visualized using an ECL kit (Beyotime, China), scanned and analyzed with TotalLab software.

Primary antibodies included: rabbit anti-FOXA1 (1:1000; Abcam), rabbit anti-ER $\alpha$ (1:2000; Epitomics) and rabbit anti$\beta$-actin (1:7500; Epitomics)

Plasmid and transfection. To stably express FOXA1, HEC-1B cells were washed with PBS and switched to antibiotic-free growth medium for $24 \mathrm{~h}$ before transfection. All transfections used Lipofectamine 2000 reagent (Invitrogen) according to the manufacturer's instructions. The plasmid pCMV6/ GFP/Neo-FOXA1 (Genechem, Shanghai, China) containing transfection-ready FOXA1 cDNA (GenBank: NM_004496) was transfected into HEC-1B cells and then the cells were selected with G418 (800 $\mu \mathrm{g} / \mathrm{ml}$; Gibco, Carlsbad, CA, USA) in the growth medium and resistant clones were chosen.

To stably silence FOXA1, Ishikawa cells were transfected with shFOXA1 (pGLV/h1/GFP/puro-FOXA1; Shanghai Genepharma Ltd., China) (5'-GAGAGAAAAAAUCAAC AGC-3') and were then selected with puromycin $(0.5 \mu \mathrm{g} / \mathrm{ml}$; Sigma Chemical, St. Louis, MO, USA). RL95-2 cells were transiently transfected with shFOXA1 in the absence of selection pressure.

For the negative controls, HEC-1B cells were transfected with a pure $\mathrm{pCMV6/GFP/Neo} \mathrm{vector,} \mathrm{and} \mathrm{Ishikawa} \mathrm{and}$
RL95-2 cells were transfected with the pGLV/h1/GFP/puro vector, respectively, under the same culture conditions.

To evaluate the effects of ER $\alpha$ expression and silencing, Ishikawa and HEC-1B cells were transiently transfected with ER $\alpha$ and shER $\alpha$ plasmids as described above, the ER $\alpha$-expressing vector (RG213277) and control vector (PS10010), the plasmid encoding ER $\alpha$ silencing short hairpin RNA (shRNA) (sh-ER $\alpha$, GI378604), and the controls (sh-NC, TR3008) (all purchased from OriGene Technologies, Beijing, China). The transfection efficiency was verified by realtime quantitative reverse-transcription PCR (qRT-PCR) and western blotting.

Cell migration assay. Cell migration activity was accomplished using Boyden chambers containing polycarbonate filters with an $8-\mu \mathrm{m}$ pore size (Millipore). Cells $\left(5 \times 10^{4}\right.$ cells/well) were resuspended with serum-free medium in the upper chamber. Medium containing $10 \%$ fetal bovine serum was added to the lower chamber. After incubation at $37^{\circ} \mathrm{C}$ for $24 \mathrm{~h}$, cells on the upper side of the membrane were removed using sterile cotton swabs. Cells adhering to the lower surface were fixed with $100 \%$ methanol and stained with hematoxylin, scanned and digital images were obtained with an Aperio Scanscope System (Aperio Technologies, USA) at a magnification of x200. Five random fields were selected for each membrane, and results are expressed in terms of the number of migratory cells per field. Each experiment was conducted in triplicate and repeated at least three times.

Cell invasion assay. Cell invasive activity was assessed using a BD BioCoat Matrigel Invasion Chamber (BD Biosciences, USA) according to the manufacturer's instructions. Cells $\left(1 \times 10^{5}\right.$ cells/well $)$ were resuspended with serum-free medium in the upper chamber with a thin layer of Matrigel matrix. Medium containing $10 \%$ FBS was added to the lower chamber. After incubation at $37^{\circ} \mathrm{C}$ for $72 \mathrm{~h}$, cells that had migrated through the membrane were fixed, stained and counted as described above.

Proliferation assay. Cells $\left(3 \times 10^{3}\right.$ cells/well) were plated in 96-well plates. Cell number was measured every $24 \mathrm{~h}$ via a colorimetric assay with 3-(4,5-dimethylthiazol-2-yl)-2, 5-diphenyltetrazolium bromide (MTT; Sigma). Absorbance at $490 \mathrm{~nm}$ was evaluated with a microplate reader (Model 680, Bio-Rad, USA). Medium was changed every other day. For the plate colony formation assay, 800 cells/well were seeded into 6-well plates. The cells were fixed and stained with crystal violet when clearly identifiable cell clones had formed. Results were detected under a light microscope. Each experiment was repeated at least three times, and assessed in triplicate.

Co-immunoprecipitation of FOXA1 and ER . Nuclear protein lysates were prepared at a concentration of $1 \mu \mathrm{g} / \mu 1$ from Ishikawa and RL95-2 EC cell lines. These lysates were incubated with $10 \mu \mathrm{g}$ anti-FOXA1 (Abcam). The immunoprecipitated proteins were collected using protein $A /$ protein $G$ agarose beads (Beyotime), washed with PBS and resuspended in loading buffer. The boiled samples were separated by SDS-PAGE, transferred to a PVDF membrane and probed with 
anti-ER $\alpha$ (Epitomics). Results were detected using an ECL kit (Beyotime), scanned, and analyzed with TotalLab software.

Tumorigenicity assays in nude mice. All experimental protocols were approved by the Ethics Committee for Animal Experimentation of Shanghai Jiao Tong University. Female BALB/C athymic nude mice 4-6 weeks old were divided into groups of six mice per group and housed with free access to food and water. To verify the effect of the silencing of FOXA1 on EC cells in vivo, we subcutaneously injected $1 \times 10^{7}$ Ishikawa cells suspended in $200 \mu 1$ 1X PBS into the unilateral foreleg of nude mice. Tumor measurement began 1 week after injection and was conducted weekly using digital calipers. Tumor volume $\left(\mathrm{mm}^{3}\right)$ was calculated using the following standard formula: Tumor volume $\left(\mathrm{mm}^{3}\right)=$ (the longest diameter) $\mathrm{x}$ (the shortest diameter) ${ }^{2}$ x 0.5 . Mice were sacrificed 5 weeks post-injection, and tumors were carefully removed and their volumes and weights were determined prior to further histological evaluation.

Statistics. Each experiment was completed at least three times, and all tests were carried out with Statistical Package for the Social Science (SPSS) software version 17.0 (Chicago, IL, USA). Data represent the mean with standard deviation (SD). Data were compared using the two-tailed Student's t-test or Mann-Whitney U-test for multiple comparisons. Differences having a probability of $\mathrm{p}<0.05$ were regarded as statistically significant.

\section{Results}

Expression of FOXA1 in tissues and its association with clinicopathological parameters. To assess whether FOXA1 is commonly upregulated in tissues, we compared its level in normal endometrial samples, atypical complex hyperplasia, and EC tissues using immunohistochemistry. Breast cancer tissues were immunostained as a positive control. The results showed that FOXA1 expression was restricted to the nucleus with little or no cytoplasmic staining (Fig. 1A). We found strong staining in normal endometrium. In contrast, staining was moderate or weak in atypical complex hyperplasia and EC tissues.

To investigate whether the change in FOXA1 expression of EC was associated with any of the clinical characteristics, we compared the association of FOXA1 expression levels with the clinicopathological parameters of the EC cases (Table I). We calculated a composite histoscore to account for both staining intensity and uniformity. Compared to the atypical hyperplasia, and EC tissues, normal endometrial tissues expressed the highest levels of FOXA1 (Fig. 1B; H-score=19.02; $<<0.001$ ). In EC tissues, strong staining of FOXA1 was observed in early-stage EC (Fig. 1A; H-score=11.35; $p=0.001$ ), whereas advanced-stage cancers had a weaker FOXA1 expression (Fig. 1A; H-score=5.00; $\mathrm{p}=0.001$ ). FOXA1 expression was also correlated with histologic type $(\mathrm{p}<0.001)$, with serous tumors $(\mathrm{H}$-score $=4.50)$ showing lower FOXA1 scores when compared with the endometrial endometrioid carcinomas (H-score=11.76). Furthermore, the status of FOXA1 was positively associated with ER levels, suggesting that FOXA1 expression has prognostic significance within the context of
ER expression $(\mathrm{p}<0.0001)$. All of these results indicate that FOXA1 is a tumor suppressor in EC, and its expression level is a favorable prognostic and diagnostic marker.

FOXA1 expression in human EC cell lines. To confirm the expression of FOXA1 in EC, 4 human EC cell lines (Ishikawa, RL95-2, HEC-1B and AN3CA) were used. Analysis of FOXA1 expression by qRT-PCR (Fig. 1C) and western blotting (Fig. 1D) revealed that there was a high endogenous FOXA1 expression in Ishikawa and RL95-2 cells, which are derived from welldifferentiated local endometrial adenocarcinoma $(16,17)$. In contrast, HEC-1B cells, which are derived from moderately well-differentiated endometrial adenocarcinoma (18) had relatively low FOXA1 expression among the cancer cell lines $(\mathrm{p}=0.0048)$.

Suppression of EC cell proliferation, migration and invasion by FOXA1. To determine whether loss of FOXA1 has an effect on EC cells, Ishikawa and RL95-2 cells were transiently transfected with a vector encoding a short hairpin RNA (shRNA) against FOXA1 and its corresponding negative control. After $48 \mathrm{~h}$, RNA was extracted and analyzed by qRT-PCR, and the protein expression was examined after $72 \mathrm{~h}$. As shown in Fig. 1E, the inhibitory efficiency of FOXA1 was $\sim 50-70 \%$ in Ishikawa and RL95-2 cells ( $<<0.01$ and $p<0.001$, respectively).

We used the MTT assay to determine whether depletion of FOXA1 induced cell proliferation; a statistically significant induction of cell proliferation was detected in two EC cell lines (Fig. 2A). The results revealed that FOXA1 depletion promoted cell proliferation in Ishikawa and RL95-2 cells in a time-dependent manner. To further explore the role of FOXA1 in cell growth, we carried out plate colony formation assays. Ishikawa and RL95-2 cells formed more colonies when transfected with shFOXA1 than with the control vector $(\mathrm{p}<0.01$; Fig. 2B). Additionally, Transwell migration (data not shown) and invasion assays (Fig. 2C) were performed to investigate the migratory and invasive potential of EC cells. FOXA1 depletion markedly promoted cell migration and invasion in Ishikawa and RL95-2 cells $(\mathrm{p}<0.001)$.

In comparison with the effect of FOXA1 knockdown, restoring FOXA1 expression in HEC-1B cells had an opposite effect. Successful reestablishment of FOXA1 expression in HEC-1B cells was confirmed by qRT-PCR and western blotting ( $<<0.001$; Fig. 1F). After $72 \mathrm{~h}$ of incubation, HEC-1B cells stably transfected with the FOXA1 expression plasmid showed significant growth inhibition when compared with the negative control cells ( $<<0.01$; Fig. 2A and B). Moreover, FOXA1 plasmid transfection decreased the migratory and invasive potential of HEC-1B cells ( $<<0.001$, Fig. 2C). All of the in vitro experiments suggest that FOXA1 plays an essential role in inhibiting EC progression.

Oncogenic role of FOXA1 in an in vivo tumor xenograft model. To further assess the role of FOXA1 in the progression of EC, we performed tumorigenicity assays in nude mice. We used shRNA-mediated stable knockdown of FOXA1 in Ishikawa cells (Fig. 3A), and the cells were injected subcutaneously. Tumors formed 1 week after injection. Over a 5-week period, we observed higher tumor growth rates in the mice injected with the cells transfected with shFOXA1 compared with the tumor 
A
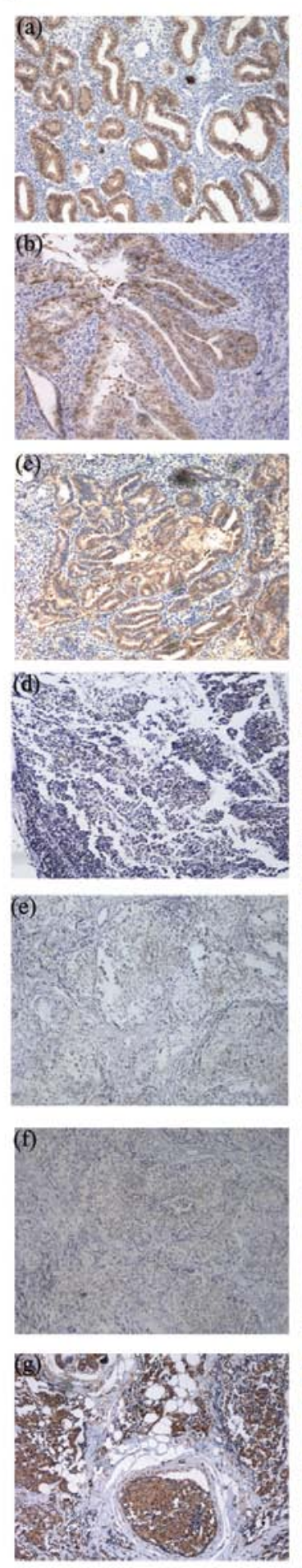

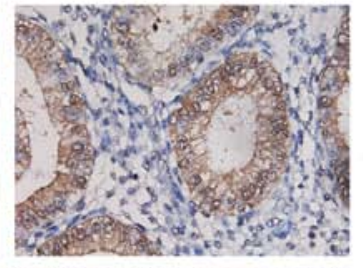

endometrium
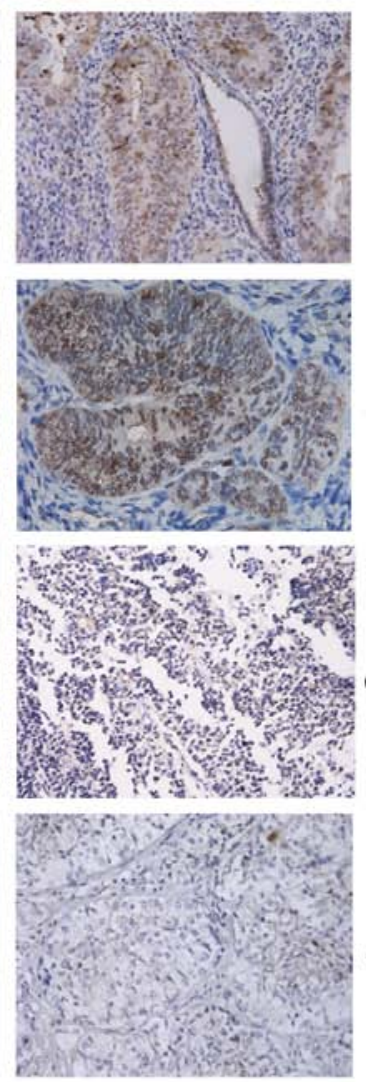

Grade 3

endometrioid

carcinoma
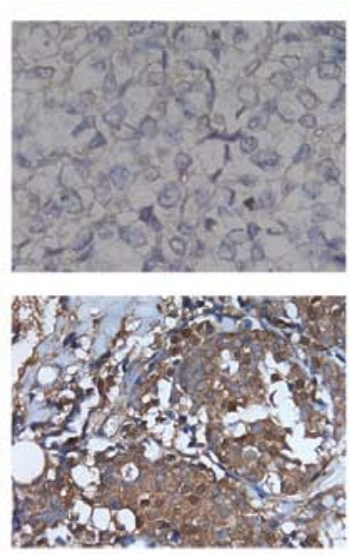

Clear cell

carcinoma

Breast
cancer

Normal

Grade 1

endometrioid

carcinoma

B

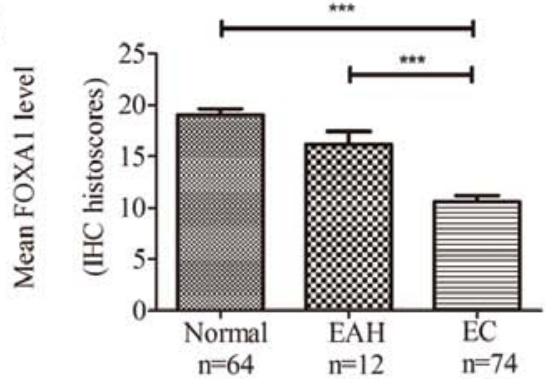

C

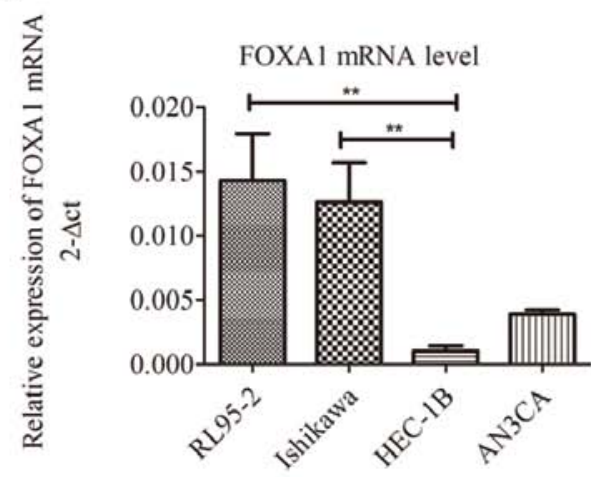

Grade 2

endometrioid

carcinoma

D

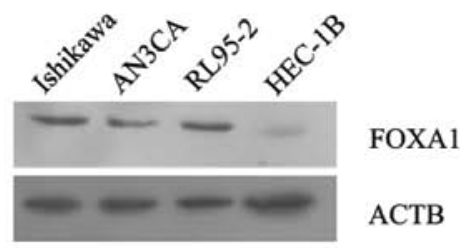

E

Ishikawa

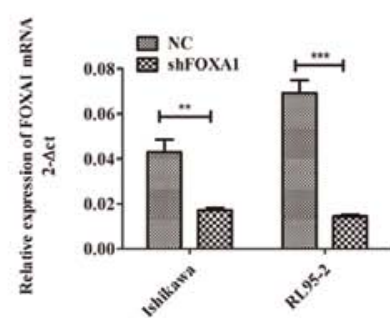

NC shFOXA1

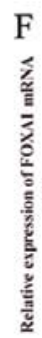
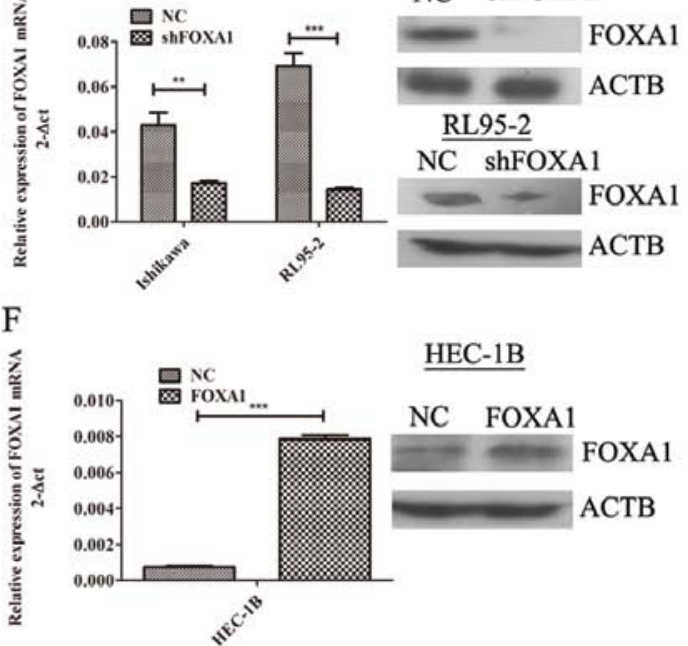

HEC-1B

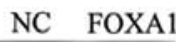

FOXA1

ACTB

Figure 1. Overexpression of FOXA1 in normal endometrium, endometrial cancer (EC) tissues and EC cell lines (A) FOXA1 immunohistochemical staining in (a) normal endometrium with strong staining, (b) atypical hyperplasia with moderate staining, (c) grade 1 endometrioid endometrial carcinoma with moderate staining, (d) grade 2 endometrioid endometrial carcinoma with weak staining, (e) grade 3 endometrioid endometrial carcinoma with weak staining, (f) clear cell carcinoma with no staining and (g) breast cancer with strong staining. Original magnification x100 (left) and x200 (right). (B) Statistical summary of the immunostaining scores in normal endometrium, endometrial atypical hyperplasia (EAH) and endometrial cancer (EC), Statistics (U-test): ${ }^{* * *} \mathrm{p}<0.001$. (C) mRNA and (D) protein expression of FOXA1 in EC cell lines as determined by qRT-PCR ( $\left.{ }^{* *} \mathrm{p}<0.01\right)$ and western blot analysis, respectively. (E) mRNA and protein expression of FOXA1 were assessed in Ishikawa and RL95-2 cells transiently transfected with shRNA against FOXA1 and its corresponding negative control. FOXA1 was downregulated after transfection. (F) mRNA and protein expression of FOXA1 was assessed in FOXA1 stable overexpressing HEC-1B cells. FOXA1 level was altered 10-fold when compared to the negative control. Data for each bar reflect triplicate measurements in each of three independent experiments. Means (bars) and SD (error bars) are shown. ${ }^{* * *} \mathrm{p}<0.01,{ }^{* * * *} \mathrm{p}<0.001$.

growth in the negative control group ( $\mathrm{p}<0.01$, Fig. 3C). Five weeks after injection, tumors were completely removed from the mice. The final mean weight and volume of the tumors were markedly higher in the shFOXA1 group than in the negative 
A

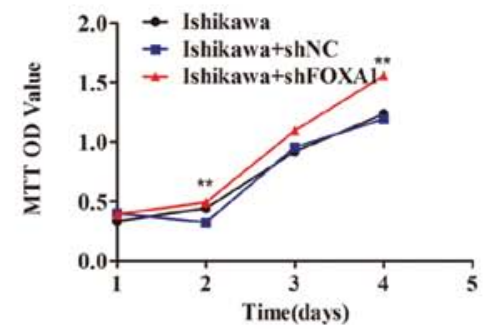

B

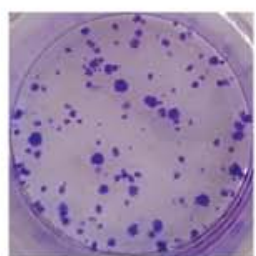

Ishikawa

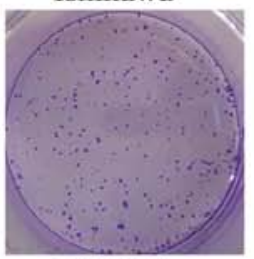

RL95-2

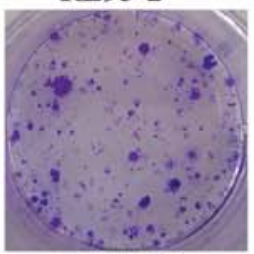

HEC-1B

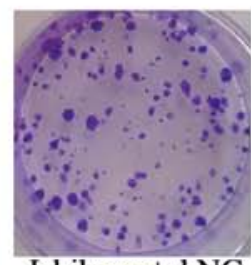

Ishikawa + shNC

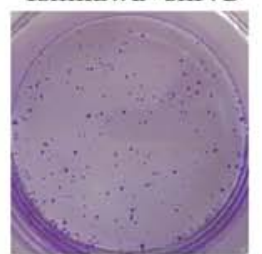

RL95-2+shNC

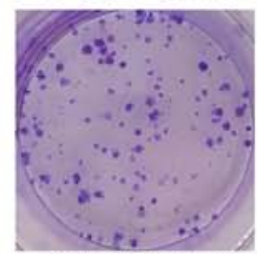

HEC-1B+NC
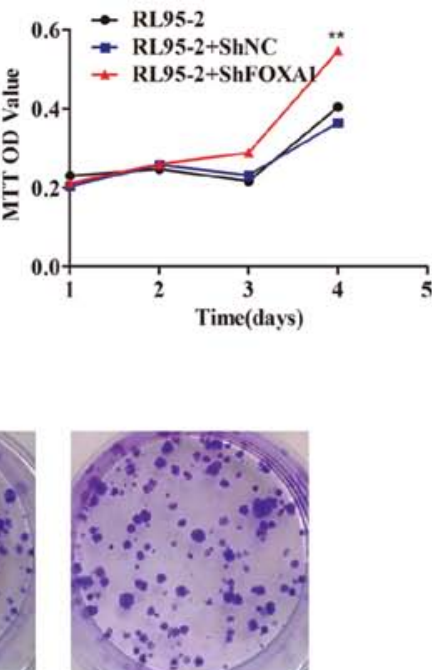

Ishikawa+shFOXA1

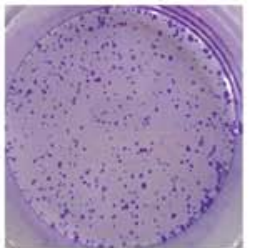

RL95-2+shFOXA1

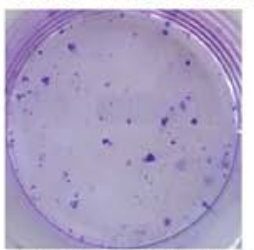

HEC-1B+FOXA1

C

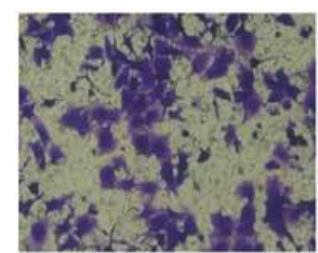

Ishikawa

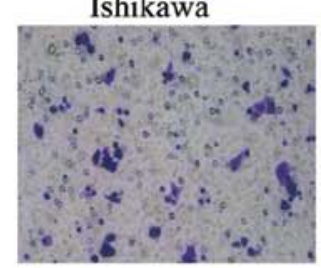

RL95-2

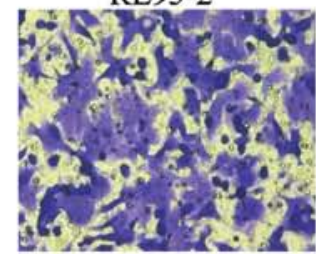

HEC-1B

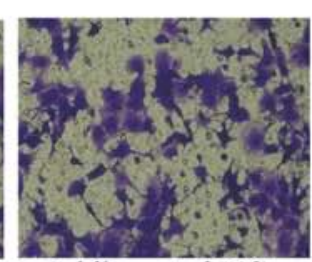

Ishikawa+shNC

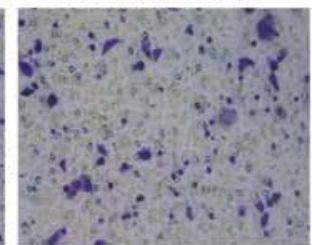

RL95-2+shNC

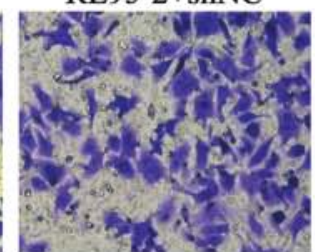

HEC-1B+NC

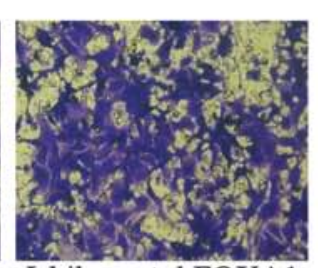

Ishikawa+shFOXA1

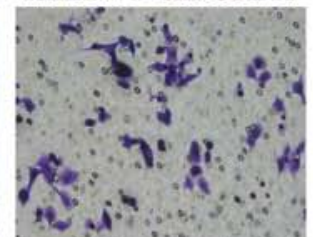

RL95-2+shFOXA1

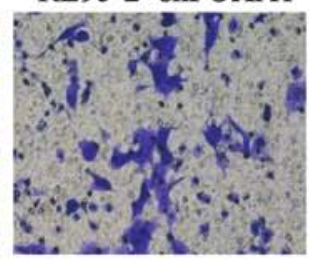

HEC-1B+FOXA1
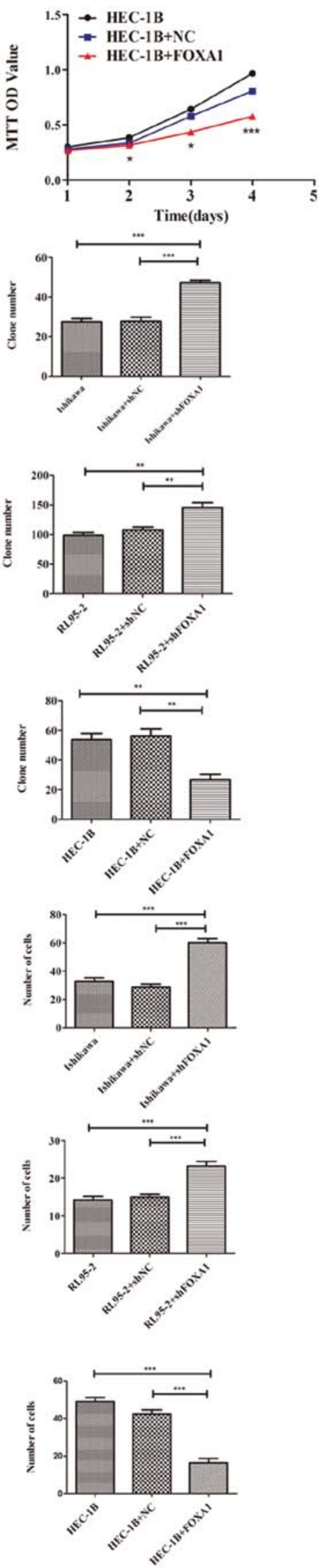

Figure 2. Effect of FOXA1 on endometrial cancer (EC) cell proliferation and invasion. (A) In vitro MTT assays were performed to examine the proliferation of Ishikawa, RL95-2 and HEC-1B cells in which FOXA1 had been knocked down. NC, negative control. (B) Plate colony formation assay was performed to observe cell proliferation of FOXA1 in Ishikawa-shFOXA1, RL95-2-shFOXA1 and HEC-1B-FOXA1 cells when compared with the negative control. (C) Representative images of Transwell assays with Matrigel coating in HEC-1B cells, which were stably transfected with the FOXA1 expression vector and vector control; Ishikawa and RL95-2 cells transiently transfected with the shFOXA1 expression vector and vector control (magnification, x200). Data for each bar reflect triplicate measurements in each of three independent experiments. Means (bars) and SD (error bars) are shown. ${ }^{* *} \mathrm{p}<0.01,{ }^{* * * *} \mathrm{p}<0.001$. 


\section{A Ishikawa shFOXA1}

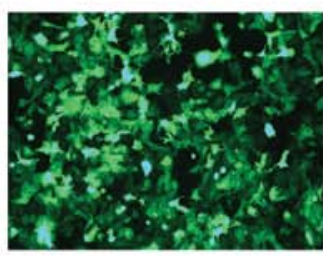

\section{B}
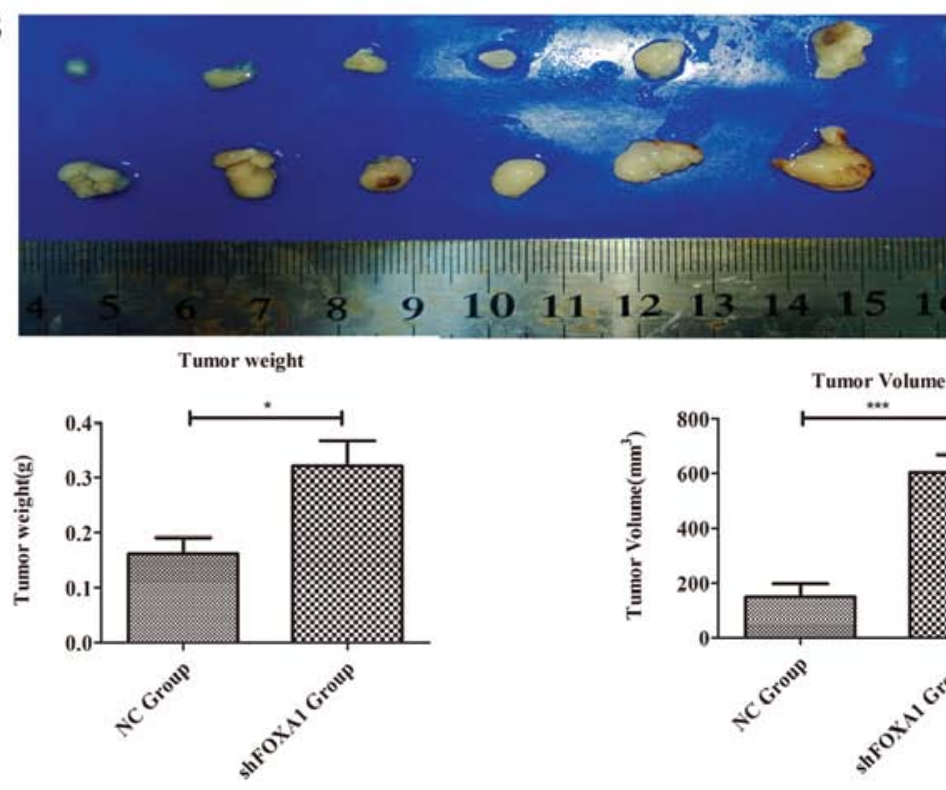

NC Group
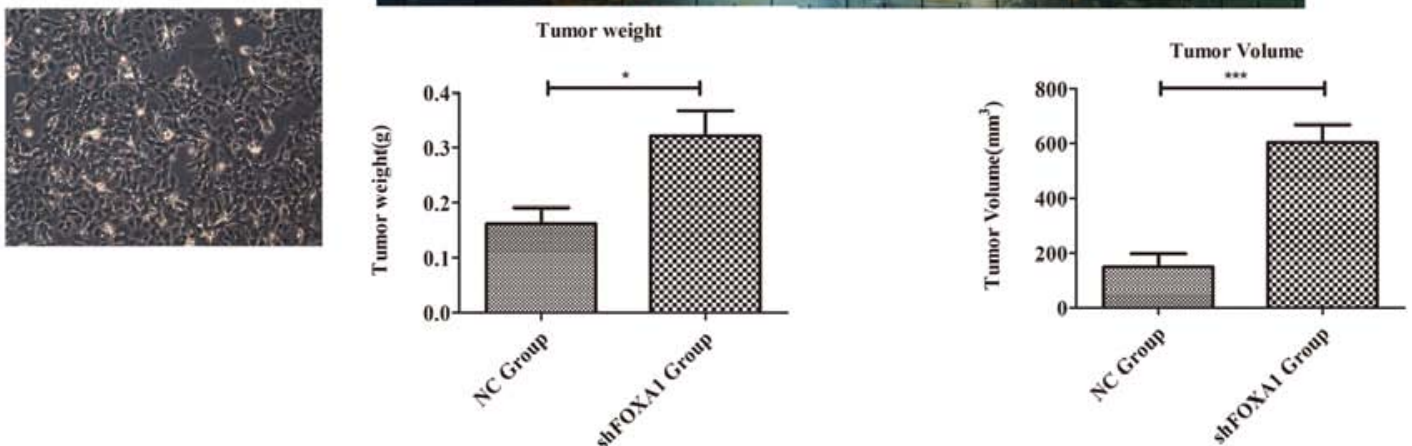

C

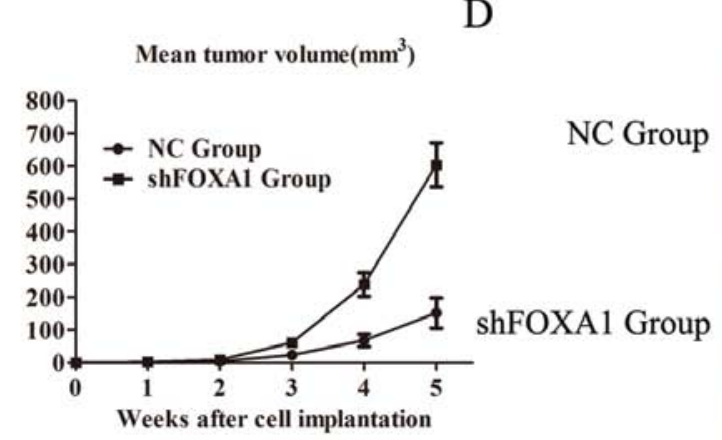

FOXA1
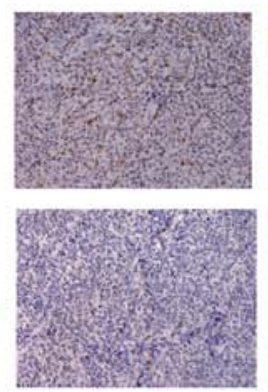

Ki67
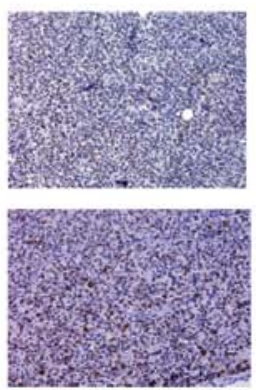

shFOXA1 Group

Figure 3. Tumorigenicity assay in nude mice. (A) Stable transfection of Ishikawa cells with shFOXA1. The efficiency of transfected cells with fluorescence was $>90 \%$ (B) The tumor formation in the nude mice. The weight and volume of the tumors were determined. Arithmetic means (bars) and SD (error bars) are shown, ${ }^{*} \mathrm{p}<0.05,{ }^{* *} \mathrm{p}<0.01,{ }^{* * *} \mathrm{p}<0.001$. (C) Tumor growth curve in the nude mice. Beginning 1 week after injection, the size of the tumor was measured weekly. (D) Tumor tissues were paraffin-embedded and stained with anti-FOXA1 (x200), anti-Ki67 (x200), and anti-ER $\alpha$ (x200).

control group ( $\mathrm{p}<0.05$ and $\mathrm{p}<0.001 ;$ Fig. 3B). To explore whether shFOXA1 influences the proliferative capacity of Ishikawa cells, we compared the levels of Ki-67 in the two groups. Previous studies revealed that expression of $\mathrm{Ki}-67$ is associated with cell proliferation (19). As shown in Fig. 3D, Ki-67 was upregulated in the shFOXA1 group $(\mathrm{p}<0.01)$. Furthermore, $\mathrm{ER} \alpha$ expression was downregulated in the shFOXA1 group $(\mathrm{p}<0.01)$. Taken together, these results suggest an important role for FOXA1 in regulating tumor viability in EC.

FOXA1 regulates the ER signaling pathway. Consistent with several reports that describe FOXA1 as a regulator of ER activity (20), our experiments used Ishikawa and RL95-2 cells as they express easily detectable levels of both FOXA1 and ER $\alpha$ (data not shown). FOXA1 knockdown by shRNA revealed a significant decrease in the expression of ER $\alpha$-dependent pS2 and GREB1 genes in the Ishikawa and RL95-2 cells in response to $10 \mathrm{nM}$ estradiol (Fig. 4A). Additionally, we found that FOXA1 depletion abolished the expression of ER $\alpha$ at the mRNA and protein levels ( $\mathrm{p}<0.05$, Fig. 4B). We also examined whether FOXA1 overexpression promotes ER $\alpha$ expression in FOXA1-overexpressing HEC-1B cells, an EC cell line with relatively low ER $\alpha$ expression. As shown in Fig. 4C, an increased FOXA1 level was correlated with higher
ER mRNA and protein levels $(\mathrm{p}<0.05)$. These results revealed that FOXA1 regulated endogenous ER $\alpha$ expression in the three EC cell lines at the post-transcriptional level. To ascertain whether ER $\alpha$ influences the FOXA1 level, we performed a reciprocal experiment. After inhibition or upregulation of the expression of $\mathrm{ER} \alpha$, we found no significant difference in FOXA1 expression (data not shown), confirming that FOXA1 acts upstream of ER $\alpha$.

We next sought to determine whether FOXA1 and ER $\alpha$ interact with each other by exploring endogenous FOXA1 and $\mathrm{ER} \alpha$ immunoprecipitates from Ishikawa cells with the reciprocal antibodies. As shown in Fig. 4D, we found an interaction between the two proteins, which demonstrated that there was a cell-specific correlation between these two genes, supporting an involvement of FOXA1 in the ER signaling pathway.

To further assess the role of FOXA1 in the estrogeninduced growth in EC cells, we transfected HEC-1B cells with a FOXA1 expression plasmid, and the proliferation assay was completed in phenol red-free medium containing $10 \%$ charcoal/dextran-treated FBS. The results showed that the EC cells expressing FOXA1 no longer proliferated following estrogen stimulation ( $p<0.001$, Fig. 4E), suggesting a functional role for FOXA1 in mediating the response to estrogen in EC. 

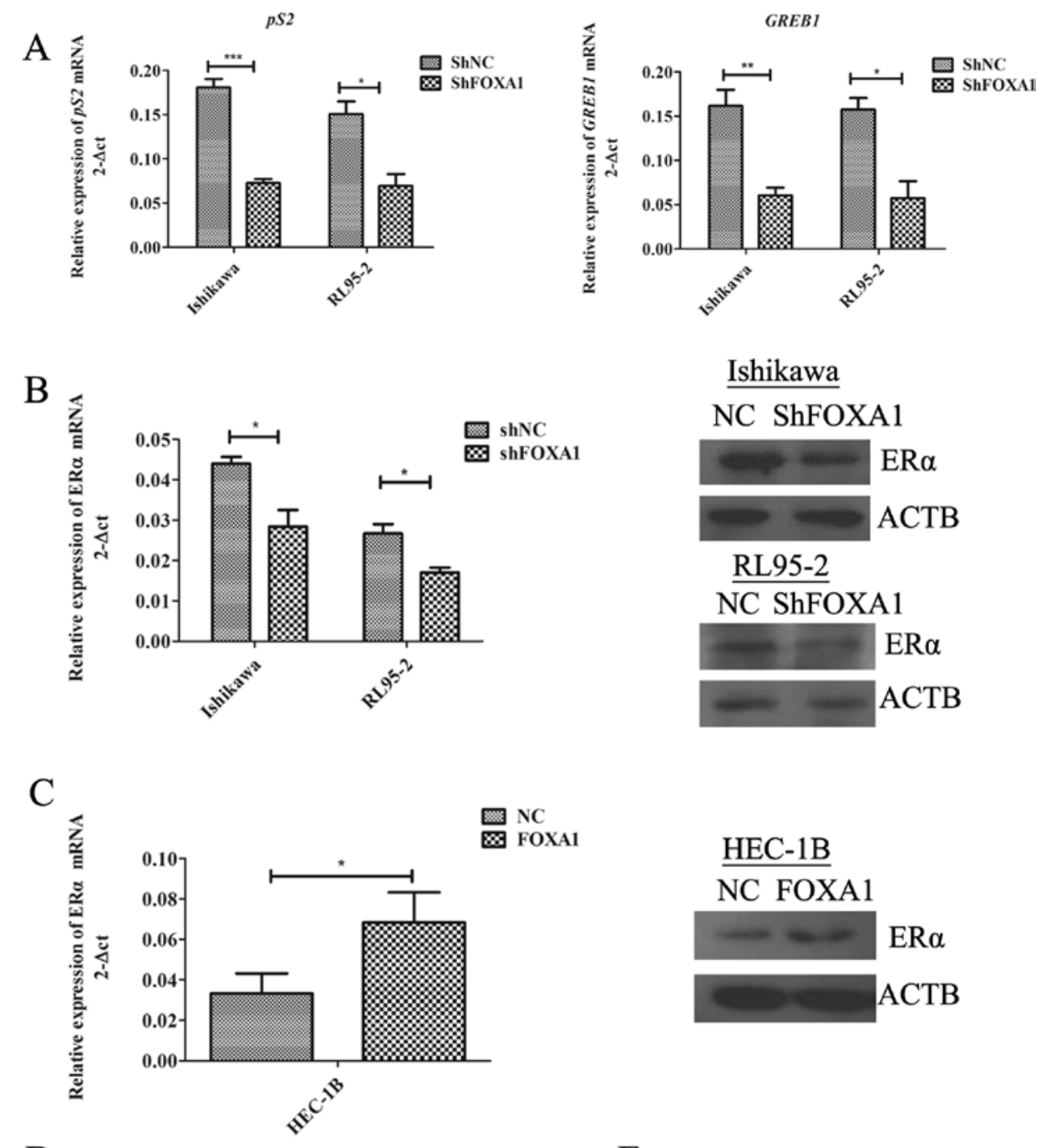

$\mathrm{D}$
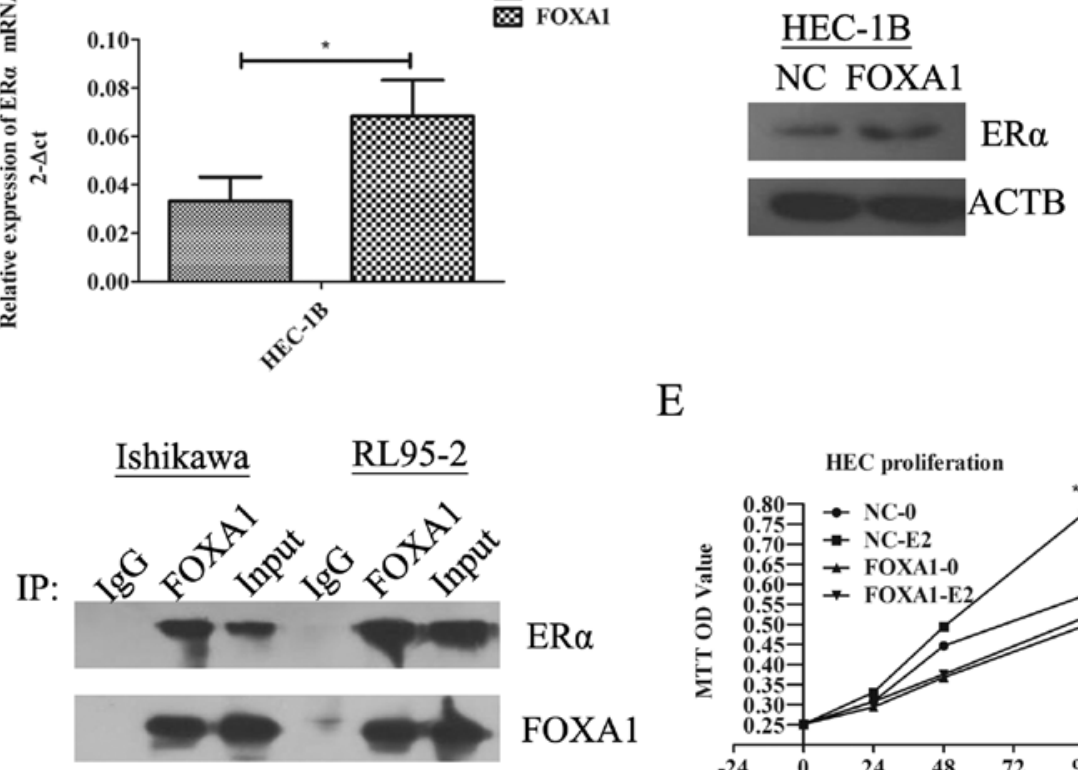

$\mathrm{E}$

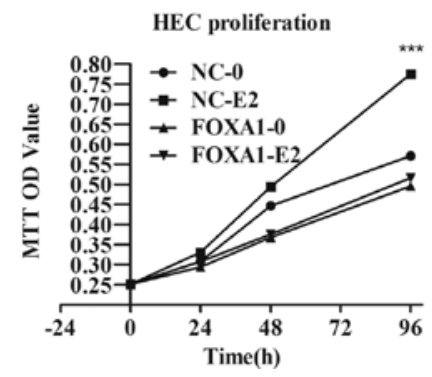

Figure 4. FOXA1 regulates the estrogen receptor signaling pathway (A) FOXA1 depletion via shRNA was accompanied by a decrease in $p S 2$ and GREB1 mRNA levels. Data for each bar reflect triplicate measurements in each of three independent experiments. Means (bars) and SD (error bars) are shown. (B) mRNA and protein expression of ER $\alpha$ was assessed in Ishikawa and RL95-2 cells transiently transfected with shFOXA1. ER $\alpha$ was downregulated after FOXA1 depletion in both cell lines. (C) mRNA and protein expression of ER $\alpha$ was assessed in FOXA1 stably overexpressing HEC-1B cells. The elevation of FOXA1 was accompanied by higher ER mRNA and protein levels. (D) Ishikawa and RL95-2 cells in serum-containing media were subject to immunoprecipitation (IP) using anti-FOXA1, anti-ER, or control antibodies prior to western blot analysis using reciprocal antibodies. (E) HEC-1B cells stably transfected with FOXA1 failed to proliferate in response to estrogen stimulation compared to control treatment. Each experiment was obtained in triplicate from three independent experiments. ${ }^{\mathrm{p}}<0.05,{ }^{* *} \mathrm{p}<0.01,{ }^{* * *} \mathrm{p}<0.001$.

\section{Discussion}

Endometrial cancer involves a heterogeneous group of tumors. Early-stage type I endometrioid tumors are often treated using adjuvant radiotherapy (21), whereas similarly staged type II serous tumors are treated with chemotherapy. Therefore, an accurate classification determines the appropriate adjuvant therapy. However, high-grade serous and endometrioid endo- metrial carcinomas are difficult to accurately subtype, and intraobserver concordance among specialized pathologists is low (22-24). Thus, the molecular classification of endometrial carcinomas may be a novel method by which to carry out informed treatment decisions.

The FOX family, particularly FOXA1, plays an important role in organ morphogenesis and disease progression (25). However, the role of FOXA1 expression or activity in EC is 
still unclear. A previous study investigated the expression pattern of FOXA1 in EC tissues, yet the samples of normal endometrium and the precise mechanism remain to be further explored (26). In the present study, we found that FOXA1 was present in almost all normal endometrial tissues, whereas differential expression was observed among the EC tumor types. We confirmed that FOXA1 can be detected in earlysatge endometrial endometrioid carcinomas more frequently than in advanced-stage cancers, including endometrioid carcinomas and serous tumors. Furthermore, the FOXA1 level was correlated with the depth of myometrial invasion and lymph node metastasis of EC, which are important prognostic factors (27). More importantly, we found a significantly positive correlation between FOXA1 and ER $\alpha$ in EC tissues, and the promotive effect of FOXA1 on ER $\alpha$ was proven in EC cell lines. This expression analysis revealed that FOXA1 was more highly expressed in well-differentiated tumors, which can provide a clinically effective prognostic factor particularly in low-risk EC patients, among ER $\alpha$-positive cases.

Over the past decade, FOXA1 expression has been reported in several types of human cancers, and the results suggest it has a dual function. Briefly, the FOXA1 level is increased in acute myeloid leukemia, esophageal cancer and lung cancer, and acts as an oncogene $(28,29)$. In contrast, in human hepatocellular carcinoma, bladder cancer, pancreatic and luminal subtype A breast cancer, FOXA1 inhibits metastasis and is a favorable prognostic factor (30-33). In the present study, we confirmed that FOXA1 overexpression in HEC-1B cells decreased cellular invasion and migration, and completely prevented estrogeninduced proliferation, whereas its depletion in Ishikawa and RL95-2 cells promoted cell viability and was associated with tumorigenicity. FOXA1 was first reported to act as a growth inhibitor through activation of the transcription of p27 in breast cancer (34). Subsequent studies revealed that FOXA1 is associated with cyclin-dependent kinase inhibitors $(13,35)$, which may explain the anti-proliferative capacity of FOXA1 in EC. In addition, our xenograft experiments showed that ablation of FOXA1 expression promoted the proliferative ability of Ishikawa cell. For several diseases, loss of FOXA1 expression promotes tumorigenesis or increases the level of tumor aggressiveness (32). All of these findings were confirmed in our study and suggest that FOXA1 acts as a tumor suppressor in EC.

An increasing number of studies suggest that crosstalk between ER and signal transduction pathways is a potential factor in the proliferation of ER $\alpha$-positive EC cells and endocrine resistance $(36,37)$. It has been well established that FOXA1 binding parallels ER $\alpha$ binding in both accessible and inaccessible regions, revealing that FOXA1 is a key determinant of ER function and endocrine response in breast cancer (20). Critically, one important issue raised by our experiments is that the expression pattern of FOXA1 in EC are strikingly similar to that of ER $\alpha$. Hence, we speculated that decreased $\mathrm{ER} \alpha$ expression (or its absence) during disease progression may be due to loss of FOXA1 function. FOXA1 depletion in vitro significantly decreased $p S 2$ and GREB1 expression, which indicates that FOXA1 is a key regulator of ER $\alpha$ gene transcription. Furthermore, specific silencing of FOXA1 was found to lead to the discrepancy of ER $\alpha$ mRNA and protein levels in the multiple EC cell lines tested. To further understand the role of FOXA1 in the ER $\alpha$ signaling pathway of EC, co-immunoprecipitation was performed and we found an interaction between the two proteins. The comparable effects of FOXA1 and ER $\alpha$ suggest that the two proteins may cooperatively regulate the transcriptional networks previously ascribed to ER $\alpha$ alone. These findings are consistent with previous studies concerning breast cancer and confirm the involvement of FOXA1 in the ER $\alpha$ signaling pathway.

A number of studies have reported that FOXA1 acts as a 'pioneer factor'. It can mimic a linker histone and can bind directly to condensed chromatin $(38,39)$. This raises the possibility that FOXA1 may provide the opportunity for other TFs to associate with chromatin. Several lines of evidence have provided an indication of the mechanistic complexity required for acute transcriptional control of steroid hormone receptors $(8,40)$. Of note, imbalanced activity of several co-regulator proteins has been proven to cause endocrine therapy-resistance, suggesting that future therapies for hormone-related diseases may target additional components of the steroid hormone receptors (41). Although our present understanding of whether FOXA1 is involved in ER $\alpha$-regulated gene transcription in endometrial cancer is limited, emerging evidence implicates an important role for FOXA1 in disease therapy (42). However, there are many TFs which may regulate the progression of endometrial cancer; therefore our team is investigating whether FOXA1 affects the function of other steroid receptors, including progesterone receptor, androgen receptor and glucocorticoid receptor, which may establish a foundation for future application of FOXA1 in the treatment of EC.

In summary, our findings confirm that FOXA1 is a valuable prognostic factor in EC, and a positive relationship exists between FOXA1 and ER $\alpha$. Further reasearch concerning FOXA1 in the steroid receptor signaling pathway for the treatment of EC is warranted.

\section{Acknowledgements}

We thank Qin Huang, Yifei Chen, and Fangyuan Wang (Centre of Research Laboratory, International Peace Maternity and Child Health Hospital Affiliated to Shanghai Jiao Tong University School of Medicine, Shanghai, China) for assisting in the preparation of this manuscript. This study was supported by the National Natural Science Foundation of China (nos. 81272885, 81172476, 81072139), the Foundation Project of Shanghai Municipal Science and Technology Commission (no. 13JC1404500) and the Ph.D. Programs Foundation of the Ministry of Education of China (no. 20120073110090).

\section{References}

1. Siegel R, Naishadham D and Jemal A: Cancer statistics, 2013. CA Cancer J Clin 63: 11-30, 2013.

2. Hannenhalli S and Kaestner KH: The evolution of Fox genes and their role in development and disease. Nat Rev Genet 10: 233-240, 2009

3. Costa RH, Grayson DR and Darnell JE Jr: Multiple hepatocyteenriched nuclear factors function in the regulation of transthyretin and alpha 1-antitrypsin genes. Mol Cell Biol 9: 1415-1425, 1989.

4. Carroll JS, Liu XS, Brodsky AS, et al: Chromosome-wide mapping of estrogen receptor binding reveals long-range regulation requiring the forkhead protein FoxA1. Cell 122: 33-43, 2005.

5. Wang Q, Li W, Zhang Y, et al: Androgen receptor regulates a distinct transcription program in androgen-independent prostate cancer. Cell 138: 245-256, 2009. 
6. Evans RM: The steroid and thyroid hormone receptor superfamily. Science 240: 889-895, 1988.

7. Vilquin P, Villedieu M, Grisard E, et al: Molecular characterization of anastrozole resistance in breast cancer: pivotal role of the Akt/mTOR pathway in the emergence of de novo or acquired resistance and importance of combining the allosteric Akt inhibitor MK-2206 with an aromatase inhibitor. Int J Cancer 133: 1589-1602, 2013.

8. Holmes KA, Hurtado A, Brown GD, et al: Transducin-like enhancer protein 1 mediates estrogen receptor binding and transcriptional activity in breast cancer cells. Proc Natl Acad Sci USA 109: 2748-2753, 2012.

9. Gaughan L, Stockley J, Coffey K, et al: KDM4B is a master regulator of the estrogen receptor signalling cascade. Nucleic Acids Res 41: 6892-6904, 2013.

10. Rahimi M, George J and Tang C: EGFR variant-mediated invasion by enhanced CXCR4 expression through transcriptional and post-translational mechanisms. Int J Cancer 126: 1850-1860, 2010.

11. Theodorou V, Stark R, Menon S and Carrol JS: GATA3 acts upstream of FOXA1 in mediating ESR 1 binding by shaping enhancer accessibility. Genome Res 23: 12-22, 2013.

12. Srijaipracharoen S, Tangjitgamol S, Tanvanich S, et al: Expression of ER, PR, and Her-2/neu in endometrial cancer: a clinicopathological study. Asian Pac J Cancer Prev 11: 215-220, 2010.

13. Wolf I, Bose S, Williamson EA, et al: FOXA1: Growth inhibitor and a favorable prognostic factor in human breast cancer. Int $\mathrm{J}$ Cancer 120: 1013-1022, 2007.

14. Creasman W: Revised FIGO staging for carcinoma of the endometrium. Int J Gynaecol Obstet 105: 109, 2009.

15. Badve S, Turbin D, Thorat MA, et al: FOXA1 expression in breast cancer - correlation with luminal subtype A and survival. Clin Cancer Res 13: 4415-4421, 2007.

16. Nishida M: The Ishikawa cells from birth to the present. Hum Cell 15: 104-117, 2002.

17. Way DL, Grosso DS, Davis JR, et al: Characterization of a new human endometrial carcinoma (RL95-2) established in tissue culture. In Vitro 19: 147-158, 1983.

18. Kuramoto H: Studies of the growth and cytogenetic properties of human endometrial adenocarcinoma in culture and its development into an established line. Acta Obstet Gynaecol Jpn 19 : 47-58, 1972.

19. Kawase M, Toyama T, Takahashi S, et al: FOXA1 expression after neoadjuvant chemotherapy is a prognostic marker in estrogen receptor-positive breast cancer. Breast Cancer: Jun 16 , 2013 (Epub ahead of print).

20. Hurtado A, Holmes KA, Ross-Innes CS, et al: FOXA1 is a key determinant of estrogen receptor function and endocrine response. Nat Genet 43: 27-33, 2011.

21. Bokhman JV: Two pathogenetic types of endometrial carcinoma. Gynecol Oncol 15: 10-17, 1983.

22. McConechy MK, Ding J, Cheang MC, et al: Use of mutation profiles to refine the classification of endometrial carcinomas. J Pathol 228: 20-30, 2012.

23. Alvarez T, Miller E, Duska L and Oliva E: Molecular profile of grade 3 endometrioid endometrial carcinoma: is it a type I or type II endometrial carcinoma? Am J Surg Pathol 36: 753-761, 2012.

24. Clarke BA and Gilks CB: Endometrial carcinoma: controversies in histopathological assessment of grade and tumour cell type. J Clin Pathol 63: 410-415, 2010.
25. Bernardo GM and Keri RA: FOXA1: a transcription factor with parallel functions in development and cancer. Biosci Rep 32: 113-130, 2012.

26. Abe Y, Ijichi N, Ikeda K, et al: Forkhead box transcription factor, forkhead box A1, shows negative association with lymph node status in endometrial cancer, and represses cell proliferation and migration of endometrial cancer cells. Cancer Sci 103: 806-812, 2012.

27. Uharcek P: Prognostic factors in endometrial carcinoma. J Obstet Gynaecol Res 34: 776-783, 2008.

28. Neben K, Schnittger S, Brors B, et al: Distinct gene expression patterns associated with FLT3- and NRAS-activating mutations in acute myeloid leukemia with normal karyotype. Oncogene 24: 1580-1588, 2005

29. Lin L, Miller CT, Contreras JI, et al: The hepatocyte nuclear factor 3 alpha gene, HNF3alpha (FOXA1), on chromosome band $14 \mathrm{q} 13$ is amplified and overexpressed in esophageal and lung adenocarcinomas. Cancer Res 62: 5273-5279, 2002.

30. Coulouarn C, Factor VM, Andersen JB, et al: Loss of miR-122 expression in liver cancer correlates with suppression of the hepatic phenotype and gain of metastatic properties. Oncogene 28: 3526-3536, 2009.

31. DeGraff DJ, Clark PE, Cates JM, et al: Loss of the urothelial differentiation marker FOXA1 is associated with high grade, late stage bladder cancer and increased tumor proliferation. PLoS One 7: e36669, 2012.

32. Song Y, Washington MK and Crawford HC: Loss of FOXA1/2 is essential for the epithelial-to-mesenchymal transition in pancreatic cancer. Cancer Res 70: 2115-2125, 2010.

33. Early Breast Cancer Trialists' Collaborative Group (EBCTCG): Effects of chemotherapy and hormonal therapy for early breast cancer on recurrence and 15-year survival: an overview of the randomised trials. Lancet 365: 1687-1717, 2005.

34. Williamson EA, Wolf I, O'Kelly J, et al: BRCA1 and FOXA1 proteins coregulate the expression of the cell cycle-dependent kinase inhibitor p27(Kip1). Oncogene 25: 1391-1399, 2006.

35. Li Q, Zhang Y, Fu J, et al: FOXA1 mediates p16(INK4a) activation during cellular senescence. EMBO J 32: 858-873, 2013.

36. Prat J, Gallardo A, Cuatrecasas M and Catasus L: Endometrial carcinoma: pathology and genetics. Pathology 39: 72-87, 2007.

37. Gururaj AE, Rayala SK, Vadlamudi RK and Kumar R: Novel mechanisms of resistance to endocrine therapy: genomic and nongenomic considerations. Clin Cancer Res 12: S1001-S1007, 2006.

38. Cirillo LA, Lin FR, Cuesta I, et al: Opening of compacted chromatin by early developmental transcription factors HNF3 (FoxA) and GATA-4. Mol Cell 9: 279-289, 2002.

39. Lupien M, Eeckhoute J, Meyer CA, et al: FoxA1 translates epigenetic signatures into enhancer-driven lineage-specific transcription. Cell 132: 958-970, 2008.

40. Kong SL, Li G, Loh SL, et al: Cellular reprogramming by the conjoint action of ERalpha, FOXA1, and GATA3 to a ligandinducible growth state. Mol Syst Biol 7: 526, 2011.

41. Green KA and Carroll JS: Oestrogen-receptor-mediated transcription and the influence of co-factors and chromatin state. Nat Rev Cancer 7: 713-722, 2007.

42. Ross-Innes CS, Stark R, Teschendorff AE, et al: Differential oestrogen receptor binding is associated with clinical outcome in breast cancer. Nature 481: 389-393, 2012. 